\title{
DETERMINATION OF LICHEN DIVERSITY VARIATIONS IN HABITAT TYPE OF MEDITERRANEAN MAQUIS AND ARBORESCENT MATORRAL
}

\author{
TUFAN-ÇETIN, Ö. \\ Program of Environmental Protection and Control, Department of Environmental Protection \\ Technology, Vocational School of Technical Sciences, University of Akdeniz, Antalya, Turkey \\ (e-mail: ozgetufan@akdeniz.edu.tr; phone: +90-242-310-6758; fax: +90-242-227-4785) \\ *Corresponding author \\ e-mail: ozgetufan@akdeniz.edu.tr \\ (Received $15^{\text {th }}$ Apr 2019; accepted $13^{\text {th }}$ Jun 2019)
}

\begin{abstract}
In this study, it was aimed to determine the epiphytic and non-epiphytic lichen diversity variations in Mediterranean maquis and arborescent matorral habitats. One of Turkey's national parks, Altınbeşik Cave National Park in Antalya, Turkey, was chosen as the research area as this habitat type is very common in the park. In this research, lichen diversity was calculated according to the data of species richness and species frequency. For identify possible variations of the lichen species composition in this habitat type, this research was planned on three major hills in the park. In addition, localities were selected from four different slope directions and tops of the hills. Statistical tests were used to analyze whether there was a difference or similarity on data. Moreover, the statistical relationships between the data of grouped localities with the data of diversity calculation classes were investigated. As a result of this study; lichen diversity of Mediterranean maquis and arborescent matorral habitats was put forth. Secondly, species richness and frequency of northern localities of Mediterranean maquis and arborescent matorral habitats were found to be significantly higher and community composition of northern localities was found to be much different than other localities.
\end{abstract}

Keywords: Altınbeşik Cave National Park, biodiversity, community composition, Eastern Mediterranean, four cardinal directions, Turkey

\section{Introduction}

Lichens are sensitive to even weak changes in their environments (Rose and Hawksworth, 1981; Ellis et al., 2007; Johansson, 2008; Nascimbene et al., 2012). Their sensitivity to environmental changes has been subject to numerous investigations (Gombert et al., 2004; Svoboda et al., 2010; De Guevara et al., 2014; Branquinho et al., 2015). When a change occurs in the environment of lichens, species richness and community composition changes to ones that are suitable for new conditions and replace previous lichen (Gadsdon et al., 2010; Johansson et al., 2012; Lang et al., 2012). This characteristic of the lichens is the reason why they are indicators of different ecosystem and habitat diversity (McCune, 2000; Rogers and Ryel, 2008; Nascimbene et al., 2012). In addition, by monitoring changes in lichen diversity, forest health can be determined (McCune, 2000; Thormann, 2006; Fenn et al., 1998; Geiser and Neitlich, 2007; McMurray et al., 2015). In order to determine the ecological condition of the areas according to the lichen diversity, it should be known which lichen composition is seen in which habitat.

Many researches have been done on lichen diversity variations of forests until now (Rogers and Ryel, 2008; McMullin et al., 2010; Koch et al., 2013; Bartels and Chen., 2015). There is also a large number of studies on the determination of the lichen richness in Mediterranean habitats (John, 1996; Litterski, 1997; Nimis and John, 1998; 
Christensen and Svane, 2007; Ravera et al., 2011; Sipman and Raus, 2015). However, there are very few studies including variations of lichen diversity in the habitat composition of Mediterranean maquis and arborescent matorral (Aragón et al., 2006).

Mediterranean maquis and arborescent matorral habitat type is found across the entire Mediterranean biogeographical zone (Portugal, Spain, France, Italy, Sardinia, Sicily, Malta, Croatia, Macedonia, Montenegro, Serbia, Bulgaria, Greece, Crete, Aegean Islands, Cyprus, Turkey and Northern Africa) (EEA, 2016). According to the habitat types of EUNIS, this habitat has evergreen sclerophilous or lauriphyllous shrub vegetation which forms a dense enclosed canopy with or without emergent trees (EEA, 2017). In this habitat type there are matorrals that are covered top to bottom with leaves that hides the stem and some of these are arborescent that are sociologically isolated, with non-competitive root system (Tomaselli, 1977). Quercus coccifera L., Pistacia palaestina (Boiss) Engl., Olea europaea L. var. sylvestris (Mill.) Brot., Phillyrea latifolia L., Juniperus excelsa M. Bieb., Pinus brutia Ten are examples of Mediterranean arborescent matorrals. Although J. excelsa and P. brutia are originally forest trees but they are also found as arborescent matorrals within maquis in Mediterranean biogeography. This interesting habitat composition may suggest that its lichen diversity is unusual.

In this study, it was aimed to determine the epiphytic and non-epiphytic lichen diversity in Mediterranean maquis and arborescent matorral habitats. Therefore, one of Turkey's national parks, Altınbeşik Cave National Park, was chosen to determine lichen diversity of Mediterranean maquis and arborescent matorral habitats of park. Besides, there is a study showing that the vegetation type of park is suitable for this research (Çinbilgel and Gökçeoğlu, 2010a). Lichen diversity in this study was calculated according to the data of species richness and species frequency. The obtained data was used not only to examine the lichen diversity in Mediterranean maquis and arborescent matorral habitats but also to examine the existing differences or similarities of lichen diversity at different directions, altitudes, and substrates (epiphytic and non-epiphytic) in this habitat type. For identify possible variations of the lichen species composition, localities were selected on three major hills (Altınbeşik Hill, İnönü Hill, Kale Hill) and from four different slope directions, tops of the hills. Moreover, the statistical relationships between the data of grouped localities (according to directions, tops and hills) with the data of diversity classes (total richness, total frequency, epiphytic and non-epiphytic richness, epiphytic and non-epiphytic frequency) were investigated.

\section{Materials and methods}

\section{Study area}

Altınbeşik Cave and surrounding area were declared as national park in 1994; located between $37^{\circ} 01.19^{\prime}$ to $37^{\circ} 04.37^{\prime} \mathrm{N}$, and $31^{\circ} 35.49^{\prime}$ to $31^{\circ} 38.52^{\prime} \mathrm{E}$ (Fig. 1). The park covers 1156 ha area, where the lowest altitude is $380 \mathrm{~m}$, and the highest is $1165 \mathrm{~m}$. The study was conducted on three major hills (Altınbeşik, İnönü and Kale) in the national park. The park is located in the rainy Mediterranean bioclimate zone according to the Emberger quotient (Çinbilgel and Gökçeoğlu, 2010b). The park generally covers with maqius and arborescent matorral habitats due to Mediterranean climate and karstic geomorphological structure of the area. Quercus coccifera L., Pistacia palaestina (Boiss) Engl., Olea europea L. var. sylvestris (Mill.) Brot., Phillyrea latifolia L., Juniperus excelsa M. Bieb., Pinus brutia Ten. are the most common members of the 
park vegetation. In addition, although the surface geology of Altınbeşik Cave National Park is generally calcareous, there are also siliceous rocks on the area.

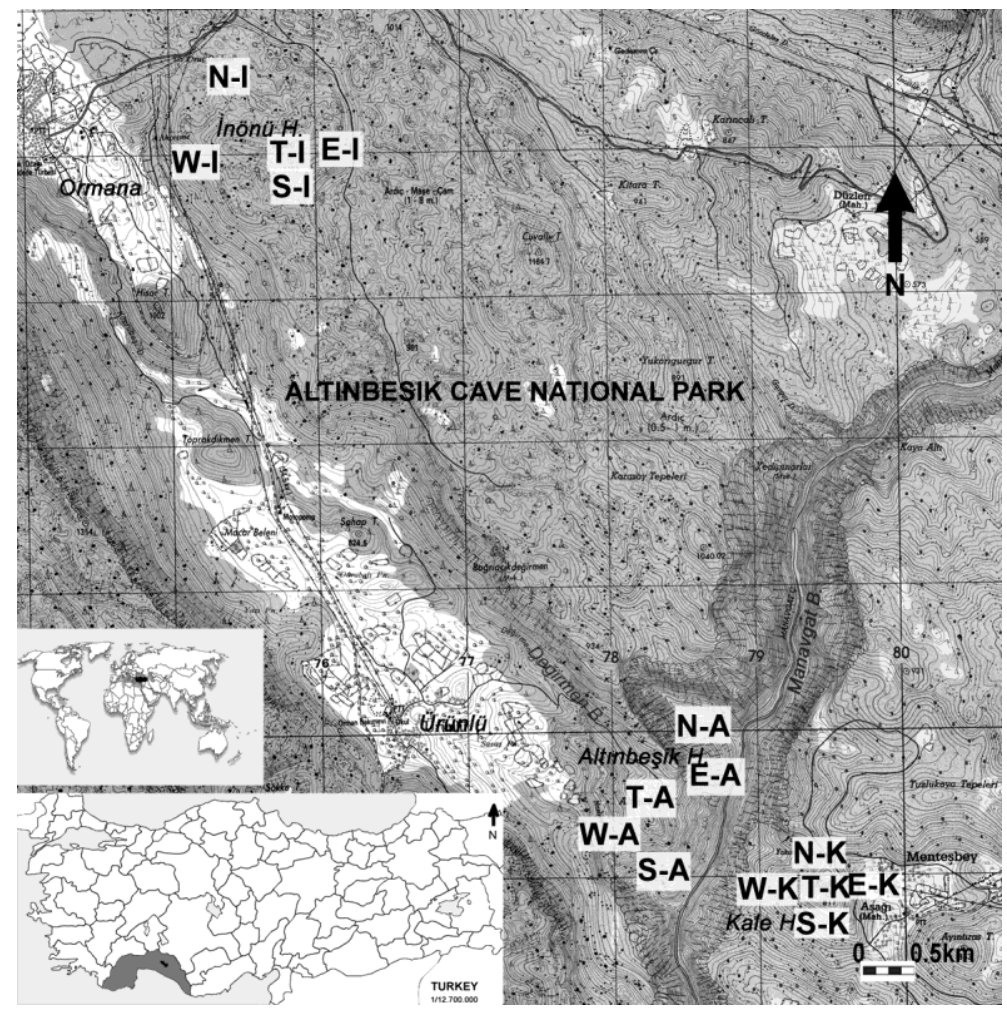

Figure 1. Maps of Altınbeşik Cave National Park and studied localities (created with Adobe Photoshop 7.0). Abbreviations: -A: Altınbeşik Hill. E: East slopes.-I: Inönü Hill. -K: Kale Hill. N: North slopes. S: South slopes. W: West slopes T: Tops

\section{Sampling and data collection techniques}

The lichen samples have been collected in April 2017, January 2019 and deposited in O. Tufan-Çetin's private fungarium in Akdeniz University. Field studies were carried out in 15 localities (sampling area approximately 1.5 ha) (Table 1; Fig. 1). The localities were selected from four different directions and tops of the 3 hills (Altınbeşik, İnönü and Kale; Fig. 1) and contain same habitat patterns (maquis and arborescent matorral habitat) and same superficial geological structure. The hills represent three different altitude groups: Altınbeşik (500-700 m), Kale (700-900 m), İnönü (900$1100 \mathrm{~m}$ ). Detail information and the abbreviated names of all localities were listed in Table 1. Sampling for richness and frequency data of lichens were recorded in $5 \times 25 \mathrm{~cm}$ quadrats with five units $(5 \times 5 \mathrm{~cm})$ for both epiphytic and non-epiphytic lichens. Frequency data was determined that based on the ratio of occurrence of the species in quadrats (Asta et al., 2002a, b). Epiphytic lichens were obtained from the trunks or stems of J. excelsa, P. latifolia, P. palaestina and Q. coccifera that found on all localities. Six individuals of each four plant species were selected and quadrats were placed $1 \mathrm{~m}$ above the ground and on four sides of the trees in each locality (Asta et al., 2002a). Also; non-epiphytic lichens from ground and rocks (calcareous and siliceous) were sampled 96 times. 
Table 1. Information of studied localities in Altınbeşik Cave National Park (İbradl, Antalya, Turkey)

\begin{tabular}{|c|c|c|c|}
\hline Abbr. & Localities & Coordinates & $\begin{array}{c}\text { Altitude } \\
\text { (Approx.) }\end{array}$ \\
\hline N-A & Manavgat Creek edges, North slopes of Altınbeşik Hill & $\begin{array}{l}37^{\circ} 02^{\prime} 18^{\prime \prime} \mathrm{N} \\
31^{\circ} 37^{\prime} 55^{\prime \prime} \mathrm{E}\end{array}$ & $585 \mathrm{~m}$ \\
\hline E-A & Entrance of Altınbeşik Cave, East slopes of Altınbeşik Hill & $\begin{array}{l}37^{\circ} 02^{\prime} 15^{\prime \prime} \mathrm{N} \\
31^{\circ} 37^{\prime} 56^{\prime \prime} \mathrm{E}\end{array}$ & $537 \mathrm{~m}$ \\
\hline S-A & South slopes of Altınbeşik Hill & $\begin{array}{l}37^{\circ} 01^{\prime} 49^{\prime \prime} \mathrm{N} \\
31^{\circ} 37^{\prime} 53^{\prime \prime} \mathrm{E} \\
\end{array}$ & $598 \mathrm{~m}$ \\
\hline W-A & West slopes of Altınbeşik Hill & $\begin{array}{l}37^{\circ} 01^{\prime} 53^{\prime \prime} \mathrm{N} \\
31^{\circ} 37^{\prime} 44^{\prime \prime} \mathrm{E} \\
\end{array}$ & $560 \mathrm{~m}$ \\
\hline T-A & Top of Altınbeşik Hill & $\begin{array}{l}37^{\circ} 02^{\prime} 05^{\prime \prime} \mathrm{N} \\
31^{\circ} 37^{\prime} 56^{\prime \prime} \mathrm{E}\end{array}$ & $653 \mathrm{~m}$ \\
\hline N-I & North slopes of İnönü Hill & $\begin{array}{l}37^{\circ} 04^{\prime} 33^{\prime \prime} \mathrm{N} \\
31^{\circ} 35^{\prime} 46^{\prime \prime} \mathrm{E}\end{array}$ & $982 \mathrm{~m}$ \\
\hline E-I & East slopes of İnönü Hill & $\begin{array}{l}37^{\circ} 04^{\prime} 22^{\prime \prime} \mathrm{N} \\
31^{\circ} 36^{\prime} 26^{\prime \prime} \mathrm{E} \\
\end{array}$ & $996 \mathrm{~m}$ \\
\hline S-I & South slopes of İnönü Hill & $\begin{array}{l}37^{\circ} 04^{\prime} 17^{\prime \prime} \mathrm{N} \\
31^{\circ} 36^{\prime} 02^{\prime \prime} \mathrm{E}\end{array}$ & $1066 \mathrm{~m}$ \\
\hline W-I & West slopes of İnönü Hill & $\begin{array}{l}37^{\circ} 04^{\prime} 21^{\prime \prime ~ N} \\
31^{\circ} 35^{\prime} 35^{\prime \prime} \mathrm{E}\end{array}$ & $912 \mathrm{~m}$ \\
\hline T-I & Top of the İnönü Hill & $\begin{array}{l}37^{\circ} 04^{\prime} 21^{\prime \prime} \mathrm{N} \\
31^{\circ} 36^{\prime} 06^{\prime \prime} \mathrm{E}\end{array}$ & $1089 \mathrm{~m}$ \\
\hline $\mathbf{N}-\mathbf{K}$ & North slopes of Kale Hill & $\begin{array}{l}37^{\circ} 01^{\prime} 40^{\prime \prime} \mathrm{N} \\
31^{\circ} 38^{\prime} 40^{\prime \prime} \mathrm{E}\end{array}$ & $747 \mathrm{~m}$ \\
\hline $\mathbf{E}-\mathbf{K}$ & East slopes of Kale Hill & $\begin{array}{l}37^{\circ} 01^{\prime} 35^{\prime \prime} \mathrm{N} \\
31^{\circ} 38^{\prime} 46^{\prime \prime} \mathrm{E} \\
\end{array}$ & $745 \mathrm{~m}$ \\
\hline S-K & South slopes of Kale Hill & $\begin{array}{l}37^{\circ} 01^{\prime} 33^{\prime \prime} \mathrm{N} \\
31^{\circ} 38^{\prime} 40^{\prime \prime} \mathrm{E}\end{array}$ & $757 \mathrm{~m}$ \\
\hline W-K & West slopes of Kale Hill & $\begin{array}{l}37^{\circ} 01^{\prime} 32^{\prime \prime} \mathrm{N} \\
31^{\circ} 38^{\prime} 32^{\prime \prime} \mathrm{E}\end{array}$ & $703 \mathrm{~m}$ \\
\hline T-K & Top of the Kale Hill & $\begin{array}{l}37^{\circ} 01^{\prime} 36^{\prime \prime} \mathrm{N} \\
31^{\circ} 38^{\prime} 41^{\prime \prime} \mathrm{E}\end{array}$ & $784 \mathrm{~m}$ \\
\hline
\end{tabular}

Abbr.: Abbreviation of localities; Approx.: Approximately

\section{Identification of samples}

Dried samples were examined by using a light microscope for microscopic characters; and a stereoscopic zoom microscope for macroscopic characters. For identification of the species, the following literature has been used: Moberg (1977), Goward et al. (1994), Fryday and Coppins (1997), Tucker and Thiers (1998), Giralt (2001), Wasser and Nevo (2005), Wetmore (2005), Smith et al. (2009), Wirth et al. (2013) and McCune (2016). When required spot tests, ultra violet light (UV) tests and thin layer chromatography (TLC) have also been carried out. 


\section{Data analysis}

The relations in recorded richness and frequency data from Altınbeşik Cave National Park were analyzed by correlation tests. First, the normality of the data was examined by the Shapiro-Wilk test. Pearson correlation test was used for normal distribution data set where Spearman's rho was used in other cases. The significance of all rankings was calculated by linear regression analysis. As of species richness and frequency data were not normally distributed, the Kruskal Wallis test (Kruskal and Wallis, 1952) was used to determine whether there is a difference within the data obtained from the localities. Since the variances were found unequal, Tamhane's T2 post-hoc test was applied to determine which localities differ from the others. All these analyses were performed by SPSS 23.0.

In terms of the lichen richness data, similarity of localities was calculated by Sørensen (Bray-Curtis) similarity index with group average algorithm (UPGMA). On the other hand, differences of community composition on maquis and arborescent matorral habitats were analyzed with non-metric multidimensional scaling (NMS) (McCune et al., 2002) using "medium" autopilot mode with the Sørensen (Bray-Curtis) distance measurement. The relationship of diversity calculation classes (joint plots) with the community composition was also investigated. Joint plots (total richness, total frequency, epiphytic and nonepiphytic richness, epiphytic and non-epiphytic frequency) were included in NMS analysis. By multi-response permutation procedure (MRPP) analysis (McCune et al., 2002), community composition of four slope directions and tops of hills, also separately three hills were compared on PC-ORD 6.08.

\section{Results}

Total 123 lichen taxa were identified from 15 selected Mediterranean maquis and arborescent matorral habitat localities in Altınbeşik Cave National Park. They are listed in Table 2 in alphabetic order. New lichen taxa for Antalya Province of Turkey are highlighted by an asterisk (*) in this list. Also, the list includes average frequencies of epiphytic and non-epiphytic taxa and their total average frequencies (total frequency) and total richness data. Total frequency and total richness data for each locality were examined and were not found normally distributed. Therefore, Spearman's rho test was used to determine whether there is any relationship between total frequency and total richness data. As a result; a strong linear relationship was found between total frequency and total richness of each localities in the positive direction $(\mathrm{p}=0.000, \mathrm{r}=0.991)$.

Based on the data of total frequency and total richness of each locality, the graph in Figure 2 was formed (The altitude of the localities are also shown in the graph). As shown in the graph, each localities were sorted from high to low total richness as follows $\mathrm{N}-\mathrm{A}>\mathrm{N}$ $\mathrm{K}>\mathrm{S}-\mathrm{A}>\mathrm{T}-\mathrm{A}>\mathrm{T}-\mathrm{K}>\mathrm{N}-\mathrm{I}>\mathrm{E}-\mathrm{I}>\mathrm{W}-\mathrm{K}>\mathrm{S}-\mathrm{I}>\mathrm{S}-\mathrm{K}>\mathrm{T}-\mathrm{I}>\mathrm{W}-\mathrm{A}>\quad \mathrm{E}-\mathrm{A}>\mathrm{W}-\mathrm{I}>\mathrm{E}-\mathrm{K}$ $(\mathrm{r}=0.868)$ and according to total frequency; from high to low: N-A $>\mathrm{N}-\mathrm{K}>\mathrm{W}-\mathrm{K}>\mathrm{S}-$ $\mathrm{A}>\mathrm{T}-\mathrm{K}>\mathrm{S}-\mathrm{I}>\mathrm{T}-\mathrm{I}>\mathrm{T}-\mathrm{A}>\mathrm{S}-\mathrm{K}>\mathrm{N}-\mathrm{I}>\mathrm{E}-\mathrm{A}>\mathrm{W}-\mathrm{I}>\mathrm{E}-\mathrm{I}>\mathrm{W}-\mathrm{A}>\mathrm{E}-\mathrm{K}(\mathrm{r}=0.852)$. In terms of total richness and frequency, the highest results were obtained from northern slopes of Altınbeşik Hill (N-A), whereas lowest results were from eastern slopes of Kale Hill (E-K). In addition, while data of N-A constitutes $16.11 \%$ of the total frequency and 21.14 of the total richness, data of E-K constitutes $2.60 \%$ of the total frequency and $10.57 \%$ of the total richness. It can be seen from the Figure 2 while the northern slopes show the highest variation in lichen diversity in maquis and arborescent matorral habitats, the eastern slopes show least. On the other hand, in terms of data of species richness and species frequency, at least one locality was found significantly different than the other localities, 
statistically (Kruskal Wallis, $\mathrm{p}=0.000$ ). Since all variances are not homogeneous, nonparametric Tamhane's T2 post-hoc test was preferred to understand which localities are different. According to richness and frequency data, especially two north localities N-A and secondly N-K are different than other 13 localities. The post-hoc test results of both localities are given at Table 3. While the similarity of localities was examined, a Sørensen dendogram based on species richness was obtained (Fig. 3). According to dendrogram, lichen richness of same slope directions is similar and also tops localities show similarity. The northern localities are in a separate similarity group the rest being in another similarity group; top localities and western localities are also in separate similarity groups.

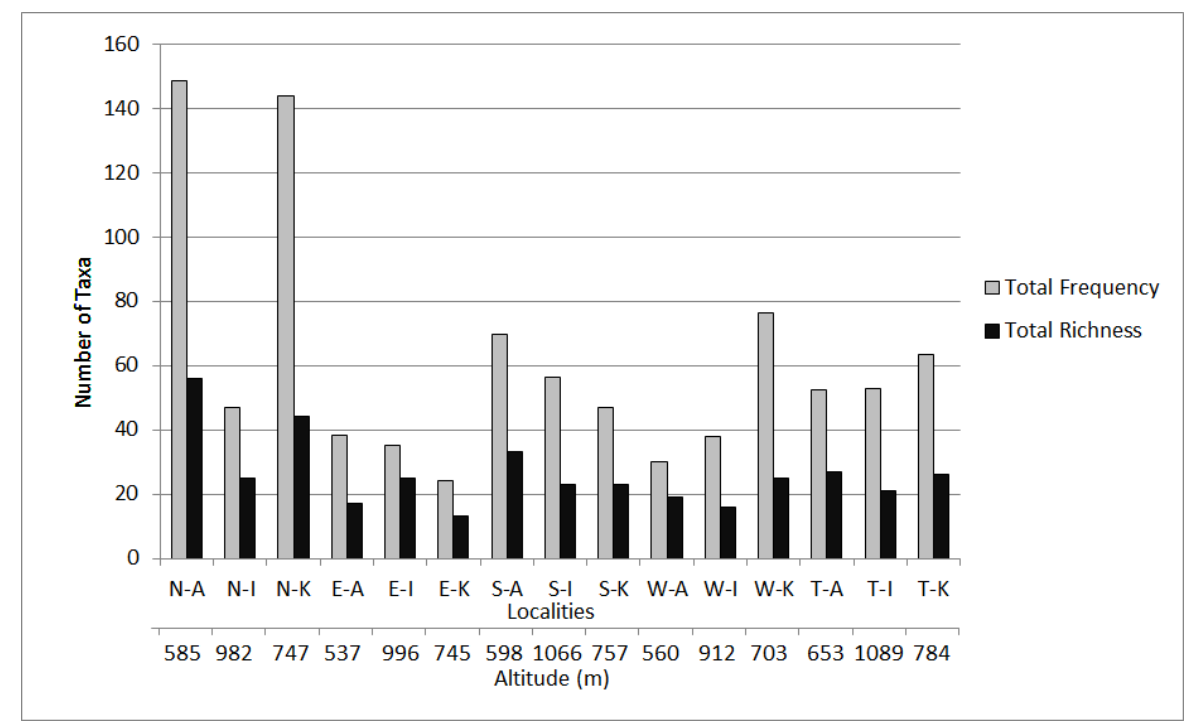

Figure 2. Graphs about total frequency and total richness data based on all localities (created with Excel 2010). Abbreviations: -A: Altınbeşik Hill. E: East slopes. -I: Inönü Hill. -K: Kale Hill. N: North slopes. S: South slopes. W: West slopes T: Tops

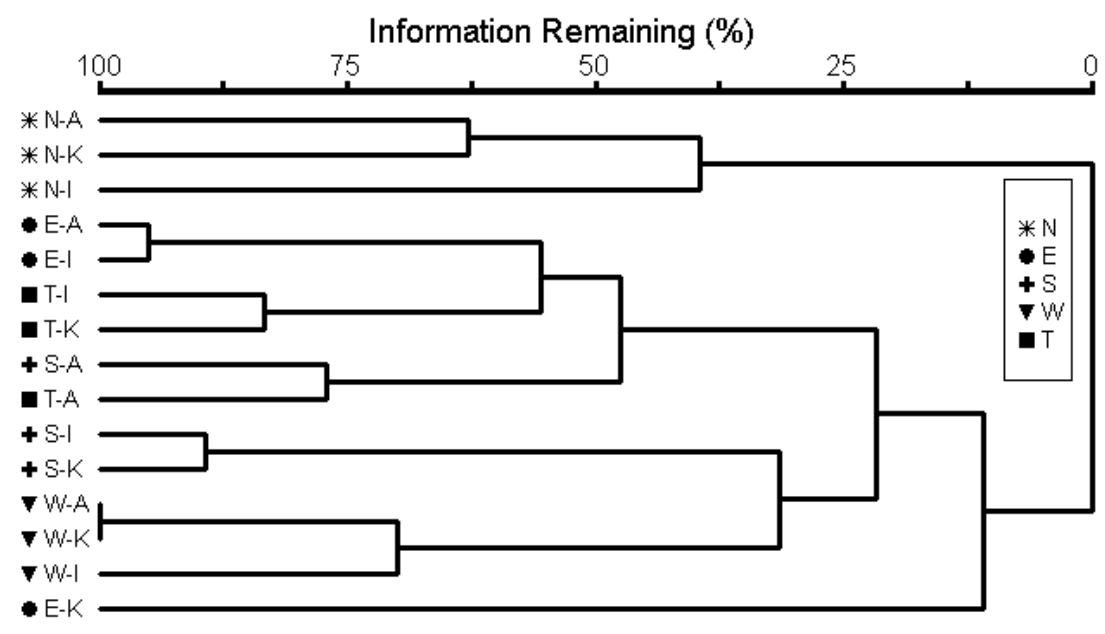

Figure 3. Sørensen similarity dendrogram of all localities based on species richness in Altınbeşik Cave National Park (created with PC-ORD 6.08). Abbreviations: -A: Altınbeşik Hill. E: East slopes.-I: Inönü Hill. -K: Kale Hill. N: North slopes. S: South slopes. W: West slopes T: 
Table 2. List of determined epiphytic and non-epiphytic lichen taxa with distribution of the average of frequencies data according to localities (the total average of frequencies and the total richness were added to the bottom of each taxa group lists)

\begin{tabular}{|c|c|c|c|c|c|c|c|c|c|c|c|c|c|c|c|c|}
\hline Epiphytic Taxa on $P$. palaestina & & N-A & N-I & N-K & E-A & E-I & E-K & S-A & S-I & S-K & W-A & W-I & W-K & T-A & T-I & T-K \\
\hline Anaptychia ciliaris (L.) Körb. ex A.Massal. & & & 4.00 & 5.67 & & & & & & & & & & & & \\
\hline Athallia cerinella (Nyl.) Arup. Frödén \& Søchting & & & & 3.67 & 0.33 & & & & & & & & & & & \\
\hline Caloplaca cerina (Ehrh. ex Hedw.) Th.Fr. & & & & 2.67 & & & & & & & & & & & 0.67 & \\
\hline Gyalolechia flavorubescens (Huds.) Søchting. Frödén \& Arup & & & & 0.33 & & & & & 3.67 & & & & & & & \\
\hline Lecanora chlarotera $\mathrm{Nyl}$ & & 1.00 & & 1.00 & & & & & 1.00 & & & & & 1.00 & & \\
\hline Lecidella elaeochroma (Ach.) M.Choisy & & & & 0.33 & & & & & & & & & & & & 1.33 \\
\hline Melanohalea exasperatula (Nyl.) O. Blanco et al. & & & & & & & & & & & & 1.00 & & & & \\
\hline Parmelina quercina (Willd.) Hale & & & & & & 0.33 & & & 3.00 & & 0.33 & & 4.00 & & & \\
\hline Phaeophyscia ciliata (Hoffm.) Moberg & & & & & & & 1.33 & & & 3.33 & & 2.00 & & & & \\
\hline Physcia leptalea (Ach.) DC. & & & & & & & & & 1.00 & & & & & & & \\
\hline Physconia distorta (With.) J.R.Laundon & & 2.00 & 3.00 & 1.33 & & & & & 3.00 & & & 3.67 & & & & \\
\hline Rinodina exigua (Ach.) Gray & & & & & & & & 2.00 & & 0.33 & & & & & & \\
\hline Xanthoria parietina (L.) Th.Fr. & & & 2.33 & 3.67 & & & & & 0.67 & & & & 1.33 & & & \\
\hline Total Frequency of Taxa & & 3.00 & 9.33 & 18.67 & 0.33 & 0.33 & 1.33 & 2.00 & 12.34 & 3.66 & 0.33 & 6.67 & 5.33 & 1.00 & 0.67 & 1.33 \\
\hline Total Richness of Taxa & & 2 & 3 & 8 & 1 & 1 & 1 & 1 & 6 & 2 & 1 & 3 & 2 & 1 & 1 & 1 \\
\hline $\begin{array}{l}\text { Epiphytic Taxa on } Q \text {. coccifera } \\
\end{array}$ & & $\mathbf{N}-\mathbf{A}$ & N-I & N-K & E-A & E-I & E-K & S-A & S-I & S-K & W-A & W-I & $\mathbf{W}-\mathbf{K}$ & T-A & T-I & T-K \\
\hline Athallia holocarpa (Hoffm.) Arup. Frödén \& Søchting & & & & & & & & 3.67 & & & & & & & & \\
\hline Caloplaca cerina (Ehrh. ex Hedw.) Th.Fr. & & & & 3.33 & & & & & & & & & & & 2.33 & \\
\hline Candelariella xanthostigma (Pers.) Lettau & & & & & & & & & & & & & & & 0.67 & \\
\hline Collema furfuraceum (Arnold) Du Rietz & & 5.67 & & & & & & 1.33 & 3.00 & & & & & & & \\
\hline Evernia prunastri (L.) Ach. & & 4.00 & & & & & & & 3.00 & 2.33 & & & & & & \\
\hline Lecanora carpinea (L.) Vain. & & & & & & & & & & & & & & 0.33 & 1.33 & 1.33 \\
\hline Lecidella elaeochroma (Ach.) M.Choisy & & 0.67 & & & 2.33 & & & & & & & & & & & \\
\hline Leptogium furfuraceum (Harm.) Sierk & & 1.33 & & & & & & & & & & & & & & \\
\hline Melanohalea exasperatula (Nyl.) O. Blanco et al. & & & & & & & & & 2.00 & & & & & & & \\
\hline Melanohalea laciniatula (Flagey ex H.Olivier) O.Blanco et al. & $*$ & 1.33 & & & & & & & & & & & & & & \\
\hline Ochrolechia balcanica Vers. & & & 0.33 & & & & & & & & & 1.33 & 2.00 & & & \\
\hline Physcia adscendens (Fr.) H.Olivier & & 5.67 & & 4.33 & & 0.67 & & & 2.00 & 2.33 & 1.33 & & 5.33 & & & \\
\hline Physcia aipolia (Ehrh. ex Humb.) Hampe & & 3.00 & & & & & & & & & & & & & & \\
\hline Physcia biziana (A.Massal.) Zahlbr. & & 1.33 & & & & & & 2.00 & 2.00 & & & & & & & \\
\hline
\end{tabular}

APPLIED ECOLOGY AND ENVIRONMENTAL RESEARCH 17(4):10173-10193

http://www.aloki.hu • ISSN 15891623 (Print) • ISSN 17850037 (Online)

DOI: http://dx.doi.org/10.15666/aeer/1704_1017310193

(๖) 2019, ALÖKI Kft., Budapest, Hungary 
Physcia leptalea (Ach.) DC.

Pleurosticta acetabulum (Neck.) Elix \& Lumbsch

Ramalina farinacea (L.) Ach.

Rinodina capensis Hamp

Xanthoria parietina $(\mathrm{L}$.) Th.Fr.

Total Frequency of Tax

Total Richness of Taxa

Epiphytic Taxa on J. excels

Candelariella efflorescens R.C.Harris \& W.R.Bucks.

Candelariella xanthostigma (Pers.) Lettau

Hypogymnia tubulosa (Schaer.) Hav.

Lecidella elaeochroma (Ach.) M.Choisy

Parmelina tiliacea (Hoffm.) Hale

Pertusaria albescens (Huds.) M.Choisy \& Werner

Polycaulina polycarpa (Hoffm.) Frödén. Arup \& Søchting

Total Frequency of Taxa

Total Richness of Taxa

Epiphytic Taxa on $P$. latifolia

Collema nigrescens (Huds.) DC.

Collema subnigrescens Degel.

Fuscopannaria olivacea (P.M.Jørg.) P.M.Jørg

Lecidella elaeochroma (Ach.) M.Choisy

Leptogium brebissonii Mont.

Leptogium cyanescens (Rabenh.) Körb.

Melanelixia glabra (Schaer.) O. Blanco et al.

Nephroma laevigatum Ach.

Parmelina tiliacea (Hoffm.) Hale

Pectenia plumbea (Lightf.) P.M.Jørg.. L.Lindblom. Wedin \& S.Ekman Pertusaria albescens (Huds.) M.Choisy \& Werner

Phaeophyscia ciliata (Hoffm.) Moberg

Phaeophyscia orbicularis (Neck.) Moberg

Phlyctis argena (Sprengel) Flot.

Physcia aipolia (Ehrh. ex Humb.) Hampe

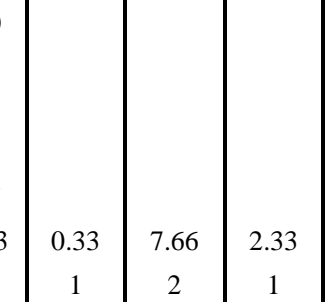

\begin{tabular}{|c|c|c|c|c|c|c|c|c|c|c|}
\hline \multirow[t]{3}{*}{0.33} & & & 1.33 & & & & \multirow[b]{3}{*}{2.00} & \multirow[b]{4}{*}{0.33} & \multirow[b]{4}{*}{4.33} & \multirow{5}{*}{$\begin{array}{c}1.33 \\
1\end{array}$} \\
\hline & & & 3.33 & & & & & & & \\
\hline & 0.67 & & 0.67 & 4.33 & 1.33 & & & & & \\
\hline 1.00 & 0.67 & 7.00 & 20.33 & 10.32 & 2.66 & 1.33 & 9.33 & & & \\
\hline 2 & 1 & 3 & 9 & 4 & 2 & 1 & 3 & 1 & 3 & \\
\hline E-I & E-K & S-A & S-I & S-K & W-A & W-I & W-K & T-A & T-I & T-K \\
\hline 0.67 & & & & & & & & 1.33 & 4.00 & \\
\hline & 0.33 & & & & & & 3.67 & 2.00 & & 0.33 \\
\hline 1.33 & & 0.67 & 2.33 & & & & & & & \\
\hline 2.00 & 0.33 & 0.67 & 2.33 & 0 & 0 & 0 & 3.67 & 3.33 & 4.00 & 0.33 \\
\hline 2 & 1 & 1 & 1 & 0 & 0 & 0 & 1 & 2 & 1 & 1 \\
\hline E-I & E-K & S-A & S-I & S-K & W-A & W-I & W-K & T-A & T-I & T-K \\
\hline & & 2.33 & & 0.67 & & & & 0.33 & & \\
\hline
\end{tabular}

\begin{tabular}{l|l|l|}
\hline & & 1.00
\end{tabular}

0.67

$\begin{array}{ll}2.00 & 6.00\end{array}$

\begin{tabular}{|l|l|l|l|}
\hline W-A & W-I & W-K & T-A \\
\hline & & & 0.33
\end{tabular}

\begin{tabular}{|l}
5.67 \\
3.00 \\
\\
\\
\\
\\
\\
\\
\\
\\
\\
4.67
\end{tabular}

2.00

3.00
1.33
1.33

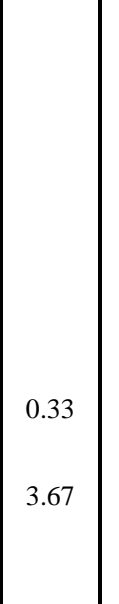


Physcia biziana (A.Massal.) Zahlbr.

Physcia leptalea (Ach.) DC.

Physconia distorta (With.) J.R.Laundon

Physconia venusta (Ach.) Poelt

Pleurosticta acetabulum (Neck.) Elix \& Lumbsch

Ramalina fastigiata (Pers.) Ach.

Total Frequency of Taxa

Total Richness of Taxa

Non-Epiphytic Taxa

Acarospora cervina (Ach.) A.Massal.

Aspicilia cheresina (Müll.Arg.) Hue

Aspicilia farinosa (Flörke) Flagey

Bagliettoa calciseda (DC.) Gueidan \& Cl.Roux

Bagliettoa marmorea (Scop.) Gueidan \& Cl.Roux

Bagliettoa parmigera (J.Steiner) Vězda \& Poelt

Blennothallia crispa (Huds.) Otálora. P.M.Jørb. \& Wedin

Caloplaca adriatica (Zahlbr.) Servit

Caloplaca atroflava (Turner) Mong

Caloplaca erythrocarpa (Pers.) Zwackh

Caloplaca velana (A.Massal.) Du Rietz

Catapyrenium daedaleum (Kremp.) Stein

Circinaria calcarea $(\mathrm{L}$.) Mudd

Circinaria contorta (L.) A. Nordin. Savić \& Tibell subsp. contorta

Circinaria contorta subsp. hoffmanniana S.Ekman \& Fröberg ex R.Sant.

Circinaria coronata (A.Massal.) B.de Lesd.

Cladonia convoluta (Lam.) Anders

Cladonia fimbriata (L.) $\mathrm{F}$

Cladonia pocillum (Ach.) Grognot

Cladonia pyxidata (L.) Hoffm.

Cladonia rangiformis Hoffm.

Clauzadea immersa (Hoffm.) Hafellner \& Bellem.

Dermatocarpon miniatum (L.) W.Mann

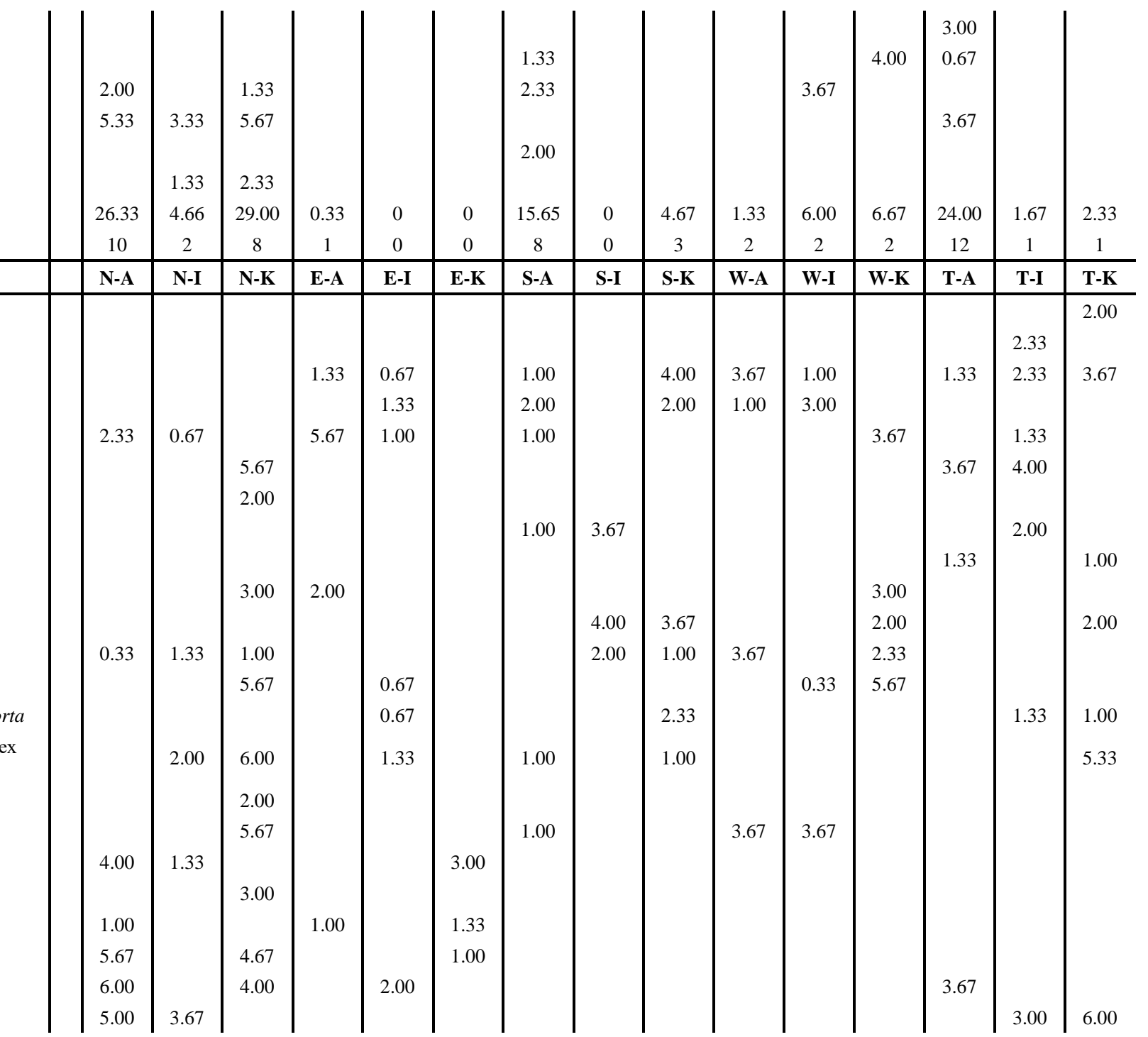

APPLIED ECOLOGY AND ENVIRONMENTAL RESEARCH 17(4):10173-10193.

http://www.aloki.hu • ISSN 15891623 (Print) • ISSN 17850037 (Online)

DOI: http://dx.doi.org/10.15666/aeer/1704_1017310193

(c) 2019, ALÖKI Kft., Budapest, Hungary 
Diploschistes ocellatus (Vill.) Norman Diplotomma venustum (Körb. in Rabenh.) Körb. Enchylium tenax (Sw.) Gray Gyalolechia flavovirescens (Wulfen) Søchting. Frödén \& Arup Gyalolechia fulgens (Sw.) Søchting. Frödén \& Arup Gyalolechia subbracteata (Nyl.) Søchting. Frödén \& Arup Lathagrium auriforme Otálora. P.M.Jørb. \& Wedin Lathagrium cristatum Otálora. P.M.Jørb. \& Wedin var. cristatum Lathagrium cristatum var. marginale (Huds.) Degel.

Lecanora bolcana (Pollini) Poelt Lecanora campestris (Schaer.) Hue Lepraria incana (L.) Ach Lepraria nivalis J.R.Laundon

Leproplaca chrysodeta (Vain. ex Räsänen) J.R.Laundon Leproplaca xantholyta (Nyl.) Hue Lobothallia radiosa (Hoffm.) Hafellner Neocatapyrenium rhizinosum (Müll.Arg.) Breuss Peltigera rufescens (Weiss) Humb. Petractis clausa (Hoffm.) Kremp. Placidium pilosellum (Breuss) Breuss Placidium squamulosum (Ach.) Breuss Placopyrenium bucekii (Nádv. \& Servít) Breuss Placynthium nigrum (Huds.) Gray Placynthium subradiatum (Nyl.) Arnold Protoparmeliopsis muralis (Schreb.) M.Choisy Psora decipiens (Hedw.) Hoffm. Psora vallesiaca (Schaer.) Timdal Pyrenodesmia chalybaea (Fr.) A.Massal. Pyrenodesmia variabilis (Pers.) A.Massal. Rhizocarpon lecanorinum Anders Rinodina calcarea (Arnold) Arnold Rinodina tunicata H.Mayrhofer \& Poelt Sarcogyne regularis Körb.

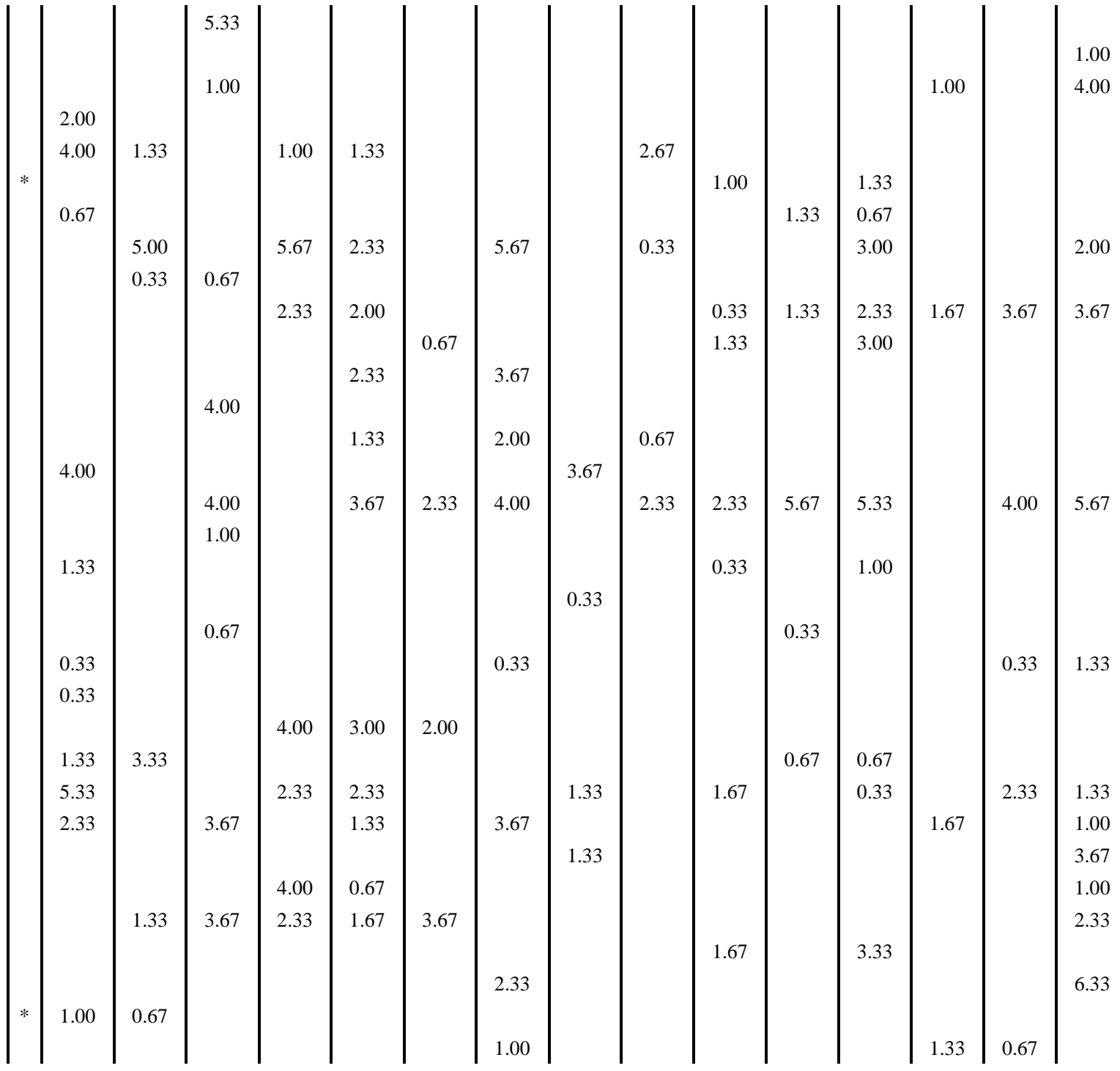

APPLIED ECOLOGY AND ENVIRONMENTAL RESEARCH 17(4):10173-10193. http://www.aloki.hu • ISSN 15891623 (Print) • ISSN 17850037 (Online) DOI: http://dx doi org/10.15666/aeer/1704_1017310193 
Scytinium gelatinosum (With.) Otálora. P.M.Jørg. \& Wedin Scytinium palmatum (Huds.) Gray

Scytinium schraderi (Bernh.) Otálora. P.M.Jørg. \& Wedin

Solenopsora cesatii (A.Massal.) Zahlbr.

Solenopsora marina (Zahlbr.) Zahlbr.

Solenopsora olivacea (Fr.) H.Kilias subsp. olbiensis (Nyl.) Clauzade \& Cl.Roux

Solenopsora olivacea (Fr.) H.Kilias subsp. olivacea

Squamarina cartilaginea (With.) P.James

Squamarina gypsacea $(\mathrm{Sm}$.) Poelt

Synalissa ramulosa (Hoffm. ex Bernh.) Fr.

Toninia candida (Weber) Th.Fr.

Toninia diffracta (A.Massal.) Zahlbr.

Toninia physaroides (Opiz) Zahlbr.

Toninia sedifolia (Scop.) Timdal

Trapelia coarctata (Turner ex Sm.) M.Choisy

Varicellaria lactea (L.) I.Schmitt \& Lumbsch

Variospora aurantia (Pers.) Arup. Frödén \& Søchting

Variospora flavescens (Huds.) Arup. Søchting \& Frödén Verrucaria dolosa Hepp

Verrucaria macrostoma Dufour ex DC.

Verrucaria nigrescens Pers.

Verruculopsis lecideoides (A.Massal.) Gueidan \& Cl.Roux

Xanthocarpia ochracea (Schaer.) A.Massal. \& De Not.

Total Frequency of Non-Epiphytic Taxa

Total Richness of of Non-Epiphytic Taxa

Total Frequency of Epiphytic Taxa

Total Richness of Epiphytic Taxa

General Total Frequency of All Taxa

General Total Richness of All Taxa 
Table 3. The most statistically different localities and multiple comparison results based on species richness and species frequency

\begin{tabular}{|c|c|c|c|c|c|}
\hline \multirow{2}{*}{\multicolumn{2}{|c|}{ Localities }} & \multicolumn{2}{|c|}{ Taxa richness } & \multicolumn{2}{|c|}{ Taxa frequency } \\
\hline & & \multirow{2}{*}{$\frac{\text { Mean difference }}{.25203^{*}}$} & \multirow{2}{*}{$\begin{array}{l}\text { Sig. } \\
.002\end{array}$} & \multirow{2}{*}{$\frac{\text { Mean difference }}{.82569^{*}}$} & \multirow{2}{*}{$\begin{array}{l}\text { Sig. } \\
.002\end{array}$} \\
\hline \multirow{14}{*}{$\mathrm{N}-\mathrm{A}$} & N-I & & & & \\
\hline & $\mathrm{N}-\mathrm{K}$ & .09756 & 1.000 & .03943 & 1.000 \\
\hline & E-A & $.31707^{*}$ & .000 & $.89602^{*}$ & .000 \\
\hline & E-I & $.25203^{*}$ & .002 & $.92309^{*}$ & .000 \\
\hline & E-K & $.34959^{*}$ & .000 & $1.01244^{*}$ & .000 \\
\hline & S-A & .18699 & .204 & .64130 & .119 \\
\hline & S-I & $.26829^{*}$ & .001 & $.74959^{*}$ & .014 \\
\hline & S-K & $.26829^{*}$ & .001 & $.82553^{*}$ & .002 \\
\hline & W-A & $.30081^{*}$ & .000 & $.96382^{*}$ & .000 \\
\hline & W-I & $.32520^{*}$ & .000 & $.89870^{*}$ & .000 \\
\hline & W-K & $.25203^{*}$ & .002 & .58699 & .446 \\
\hline & $\mathrm{T}-\mathrm{A}$ & $.23577^{*}$ & .008 & $.78211^{*}$ & .006 \\
\hline & T-I & $.28455^{*}$ & .000 & $.77943^{*}$ & .008 \\
\hline & $\mathrm{T}-\mathrm{K}$ & $.24390^{*}$ & .004 & 69285 & .076 \\
\hline \multirow{14}{*}{$\mathrm{N}-\mathrm{K}$} & N-I & -.09756 & 1.000 & -.03943 & 1.000 \\
\hline & $\mathrm{N}-\mathrm{K}$ & .15447 & .516 & $.78626^{*}$ & .007 \\
\hline & E-A & $.21951^{*}$ & .006 & $.85659^{*}$ & .002 \\
\hline & E-I & .15447 & .516 & $.88366^{*}$ & .000 \\
\hline & E-K & $.25203^{*}$ & .000 & $.97301^{*}$ & .000 \\
\hline & S-A & .08943 & 1.000 & 60187 & .278 \\
\hline & S-I & .17073 & .234 & $.71016^{*}$ & .043 \\
\hline & S-K & .17073 & .234 & $.78610^{*}$ & .007 \\
\hline & W-A & $.20325^{*}$ & .024 & $.92439^{*}$ & .000 \\
\hline & W-I & $.22764^{*}$ & .003 & $.85927^{*}$ & .002 \\
\hline & W-K & .15447 & .516 & .54756 & .706 \\
\hline & T-A & .13821 & .829 & $.74268^{*}$ & .020 \\
\hline & T-I & .18699 & .083 & $.74000^{*}$ & .026 \\
\hline & $\mathrm{T}-\mathrm{K}$ & .14634 & .682 & .65341 & .183 \\
\hline
\end{tabular}

*The mean difference is significant at the 0.05 level

After Kruskal Wallis analyses were used, at least one locality was found significantly different than the other localities. Since all variances are not homogeneous, non-parametric Tamhane's T2 post-hoc test was preferred

-A: Altınbeşik Hill, -I: İnönü Hill, -K: Kale Hill, E: East slopes, N: North slope, S: South slopes, W: West slopes, T: Tops

After these results were determined, total frequency and total richness data were grouped based on localities at different slope directions and tops of the hills (Fig. 4). Also; localities from three hills grouped separately and plotted accordingly (Fig. 5). As two comparison data showed normal distribution, Pearson test was used for correlation analysis of these. While there was a very strong linear relation in data of four slope directions and data of tops $(p=0.008, r=0.963)$, no relation was found within the data of the three hills (and the three altitude groups). As seen from the graphs, the total 
frequency of different slope directions and tops is sorted from high to low as follows: North $>$ South $>$ Top $>$ West $>$ East $(r=0.885)$; total richness data as North $>$ South $>$ Top $>$ East $>$ West $(r=0.911)$. Total northern data were found to be significantly higher than other directions and tops (Fig. 4).

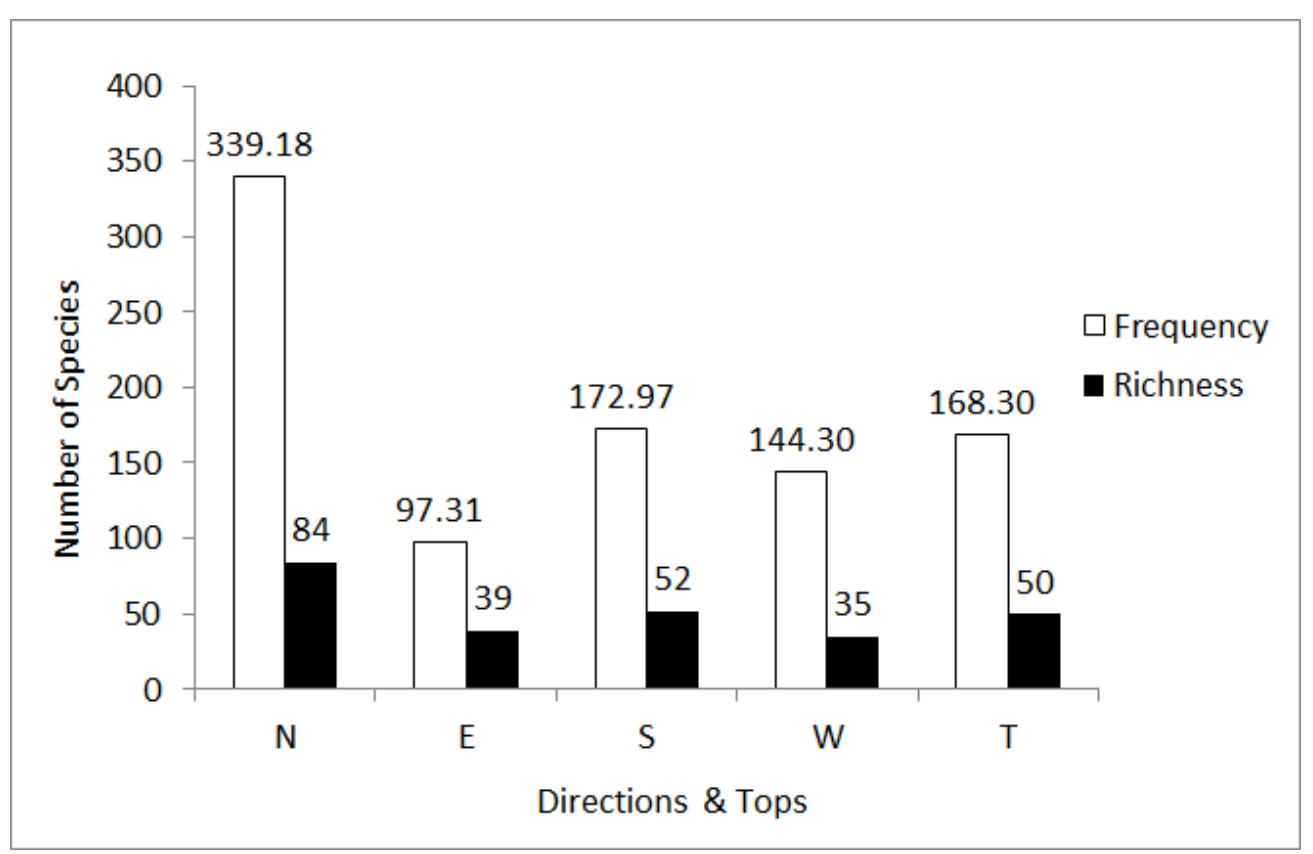

Figure 4. Graphs about total frequency and total richness data based on localities of different slope directions and tops (created with Excel 2010). Abbreviations: E: East slopes, N: North slopes, S: South slopes, W: West slopes, T: Tops

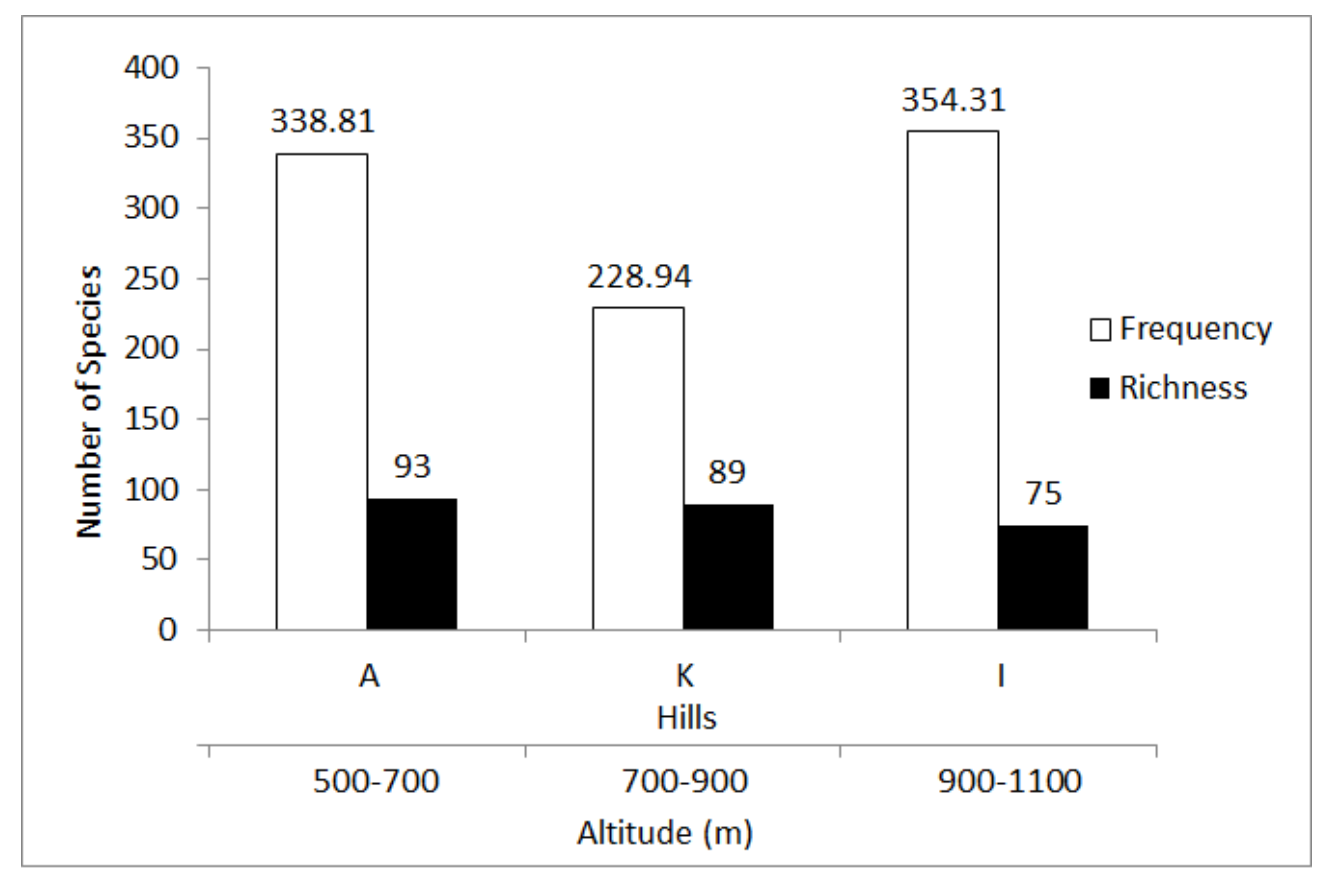

Figure 5. Graphs about total frequency and total richness data based on localities of three hills (created with Excel 2010). Abbreviations: A: Altınbeşik Hill, I: İönü Hill, K: Kale Hill 
Also; total frequency data results obtained from the three hills are as follows from high to low (These hills also represent different altitude groups): Altınbeşik (500$700 \mathrm{~m})>$ Kale $(700-900 \mathrm{~m})>$ İnönü $(900-1100 \mathrm{~m})(\mathrm{r}=0.917)$; total richness data as: İnönü $(900-1100 \mathrm{~m})>$ Altınbeşik $(500-700 \mathrm{~m})>$ Kale $(700-900 \mathrm{~m})(\mathrm{r}=0.952)$ (Fig. 5). When Kruskal Wallis test was applied for data of species richness and species frequency of different slope directions and tops, at least one data group statistically differentiates from others $(\mathrm{p}=0.000)$. In addition, according to Tamhane's T2 post-hoc test results; northern slopes data significantly differ from the data of other directions and tops (Table 4). When species richness and frequency data of the three hills were analyzed; difference is available for the frequency data (Kruskal Wallis, $p=0.033$ ) and richness data (Kruskal Wallis, $\mathrm{p}=0.011$ ) of the three hill. Tamhane's T2 post-hoc test result showed; Inönü Hill (900-1100 m) is different from Altınbeşik Hill (500-700 m) based on species richness data and it is different from Altınbeşik and Kale (700-900 m) Hills based on frequency data (Table 5).

Table 4. Multiple comparison results based on species richness and species frequency of data of slope directions and tops

\begin{tabular}{|c|c|c|c|c|c|}
\hline \multirow{2}{*}{\multicolumn{2}{|c|}{ Directions \& tops }} & \multicolumn{2}{|c|}{ Species richness } & \multicolumn{2}{|c|}{ Species frequency } \\
\hline & & \multirow{2}{*}{$\begin{array}{c}\text { Mean difference } \\
.36585^{*}\end{array}$} & \multirow{2}{*}{$\begin{array}{l}\text { Sig. } \\
.000\end{array}$} & \multirow{2}{*}{$\begin{array}{c}\text { Mean difference } \\
1.97724^{*}\end{array}$} & \multirow{2}{*}{$\begin{array}{l}\text { Sig. } \\
.000\end{array}$} \\
\hline \multirow{4}{*}{$\mathrm{N}$} & $\mathrm{E}$ & & & & \\
\hline & S & $.26016^{*}$ & .000 & $1.36211^{*}$ & .001 \\
\hline & W & $.39837^{*}$ & .000 & $1.59520^{*}$ & .000 \\
\hline & $\mathrm{T}$ & $.27642^{*}$ & .000 & $1.40008^{*}$ & .001 \\
\hline \multirow{4}{*}{$\mathrm{E}$} & $\mathrm{N}$ & $-.36585^{*}$ & .000 & $-1.97724^{*}$ & .000 \\
\hline & S & -.10569 & .596 & -.61512 & .111 \\
\hline & W & .03252 & 1.000 & -.38203 & .790 \\
\hline & $\mathrm{T}$ & -.08943 & .793 & -.57715 & .219 \\
\hline \multirow{4}{*}{$S$} & $\mathrm{~N}$ & $-.26016^{*}$ & .000 & $-1.36211^{*}$ & .001 \\
\hline & $\mathrm{E}$ & .10569 & .596 & .61512 & .111 \\
\hline & $\mathrm{W}$ & .13821 & .211 & .23309 & .994 \\
\hline & $\mathrm{T}$ & .01626 & 1.000 & .03797 & 1.000 \\
\hline \multirow{4}{*}{ W } & $\mathrm{N}$ & $-.39837^{*}$ & .000 & $-1.59520^{*}$ & .000 \\
\hline & $\mathrm{E}$ & -.03252 & 1.000 & .38203 & .790 \\
\hline & S & -.13821 & .211 & -.23309 & .994 \\
\hline & $\mathrm{T}$ & -.12195 & .366 & -.19512 & .999 \\
\hline \multirow{4}{*}{$\mathrm{T}$} & $\mathrm{N}$ & $-.27642^{*}$ & .000 & $-1.40008^{*}$ & .001 \\
\hline & E & .08943 & .793 & .57715 & .219 \\
\hline & S & -.01626 & 1.000 & -.03797 & 1.000 \\
\hline & $\mathrm{W}$ & .12195 & .366 & .19512 & .999 \\
\hline
\end{tabular}

*The mean difference is significant at the 0.05 level

After Kruskal Wallis analysis were used. At least one slope direction or top data was found significantly different than the other localities. Since all variances are not homogeneous. non-parametric Tamhane's T2 post-hoc test was preferred

E: East slopes. N: North slopes. S: South slopes. W: West slopes T: Tops 
Table 5. Multiple comparison results based on species richness and species frequency of data of three hills

\begin{tabular}{c|c|c|c|c|c}
\hline \multicolumn{2}{c|}{ Hills } & \multicolumn{2}{c|}{ Species richness } & \multicolumn{2}{c}{ Species frequency } \\
\cline { 3 - 6 } \multicolumn{2}{c|}{} & Mean difference & Sig. & Mean difference & Sig. \\
\hline \multirow{2}{*}{ A } & I & $.14634^{*}$ & .040 & $.90407^{*}$ & .030 \\
& $\mathrm{~K}$ & .03252 & .916 & -.11520 & .989 \\
\hline \multirow{2}{*}{$\mathrm{I}$} & $\mathrm{A}$ & $-.14634^{*}$ & .040 & $-.90407^{*}$ & .030 \\
& $\mathrm{~K}$ & -.11382 & .166 & $-1.01927^{*}$ & .021 \\
\hline \multirow{2}{*}{$\mathrm{K}$} & $\mathrm{A}$ & -.03252 & .916 & .11520 & .989 \\
& $\mathrm{I}$ & .11382 & .166 & $1.01927^{*}$ & .021 \\
\hline
\end{tabular}

*The mean difference is significant at the 0.05 level

After Kruskal Wallis analysis were used. At least one hill data was found significantly different than the other localities. Since all variances are not homogeneous. non-parametric Tamhane's T2 post-hoc test was preferred

A: Altınbeşik Hill, I: İnönü Hill, K: Kale Hill

When species community composition was summarized according to frequency data by non-metric multidimensional scaling (NMS), it was seen that 3 axes explained total variation with $82.89 \%$ (axis $148.20 \%$; axis $221.81 \%$; axes $312.88 \%$ ). For 3 dimensional solution, the final stress rate was $12.41 \%$ and instability was 0.00000 . At Figure 6, an ordination graph was given based on these NMS results with joint plot vectors (total richness, total frequency, epiphytic and non-epiphytic richness, epiphytic and non-epiphytic frequency). Examining Pearson correlation of axes and vectors; it was found that total richness $(r=0.624)$, epiphytic richness $(r=0.610)$, non-epiphytic richness $(r=0.470)$, epiphytic frequency $(r=0.552)$, non-epiphytic frequency $(\mathrm{r}=0.462)$ and total frequency $(\mathrm{r}=0.556)$ showed high positive correlations with axis 1. At axis 2; whereas all diversity vectors had descending relation, epiphytic richness $(\mathrm{r}=0.708)$ and epiphytic frequency $(\mathrm{r}=0.715)$ were associated with ascending gradient. It can be understood from the results and graph; the epiphytic richness and frequency are more related to the data from southern slopes than data from the top and other slopes. Furthermore, while all other vector parameters were associated with the northern slopes, Figure 6 shows non-epiphytic richness and frequency are also associated with the data of tops of hills.

Lichen community composition of tops and four slope directions of hills were compared as small-scale with MRPP. The results were presented in Table 6. In a MRPP analysis, in case chance-corrected within-group agreement $(A)$ value is higher than 0.3 , this $A$ value is enough to say the compositions are different (McCune et al., 2002). When the results of analysis were evaluated according to this information, species composition of northern slopes versus southern, eastern, western slopes and tops were found distinctly different as well as western slopes versus tops (Table 6). On NMS graph, also MRPP results are reflected (Fig. O). Also it was experimented comparison of lichen community composition as large-scale with the data of three hills. In Table 6, MRPP comparison of lichen community composition of three hills is given separately. According to this table, no significant difference is observed in any pairwise comparison. 


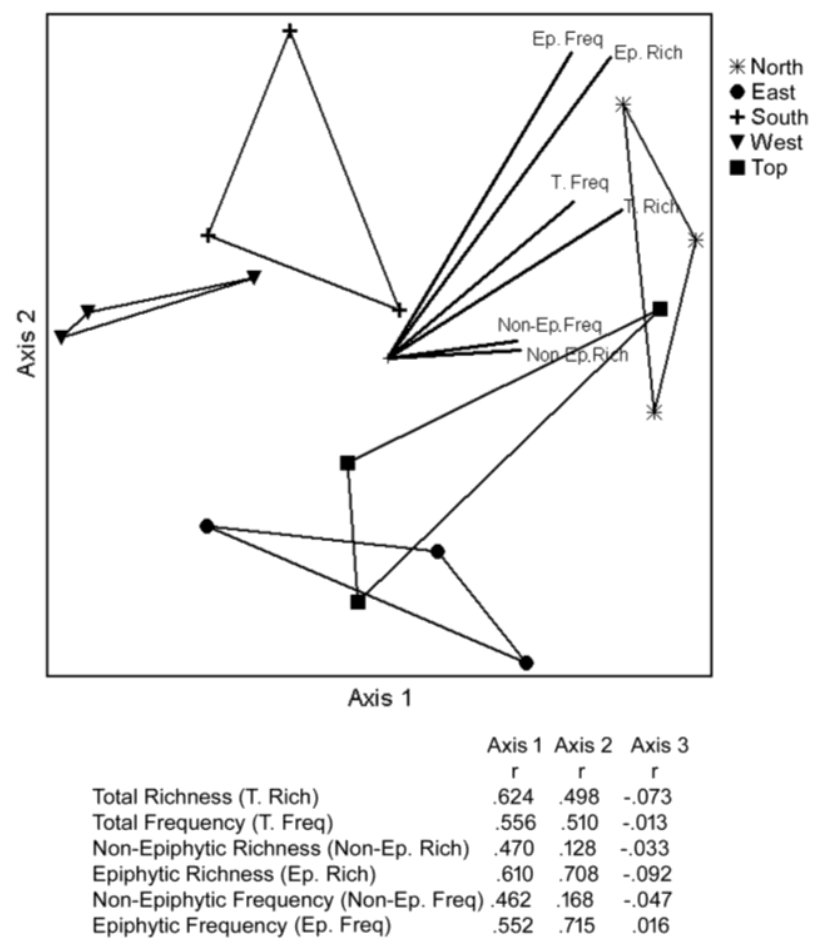

Figure 6. Ordination graph based on NMS results with diversity calculation classes (joint plot vectors) (created with PC-ORD 6.08)

Table 6. Pairwise comparison of lichen community composition of tops-four slope directions and separately hills with MRPP analysis

\begin{tabular}{c|c|c}
\cline { 2 - 3 } & \multicolumn{2}{c}{ Directions \& tops } \\
\cline { 2 - 3 } N vs. E & 0.41 & $\boldsymbol{p}$ (MRPP) \\
N vs. S & 0.38 & 0.02345 \\
N vs. W & 0.43 & 0.02852 \\
N vs. T & 0.30 & 0.02278 \\
E vs. S & 0.24 & 0.04784 \\
E vs. W & 0.27 & 0.05580 \\
E vs. T & 0.11 & 0.04174 \\
S vs. W & 0.19 & 0.16028 \\
S vs. T & 0.25 & 0.08800 \\
W vs. T & 0.33 & 0.03982 \\
& \multicolumn{2}{|c}{ Hills } \\
\hline & $\boldsymbol{A}$ (MRPP) & 0.02374 \\
\hline A vs. I & -0.087 & $\boldsymbol{p}$ (MRPP) \\
A vs. K & 0.014 & 0.8898 \\
I vs. K & -0.107 & 0.3834 \\
\hline A & & 0.9607 \\
\hline
\end{tabular}

A value is higher than 0.3 , this A value is enough to say the compositions are different (McCune et al., 2002)

A: Altınbeşik Hill, A (MRPP): Chance-corrected within-group agreement, E: East slopes, I: İnönü Hill, K: Kale Hill, N: North slopes, p (MRPP): calculated probability, S: South slopes, vs.: versus, W: West slopes, T: Tops 


\section{Discussion}

The study was carried out to determine the compositional diversity of lichen species in selected Mediterranean maquis and arborescent matorral habitats in Altınbeşik Cave National Park. Nascimbene et al. (2013) listed 35 articles examining lichen richness, diversity and composition of different habitats in different European countries. These articles were based on epiphytic lichens that live on forest trees. This study differentiates from those articles by examining epiphytic and non-epiphytic lichens on maquis and arborescent matorral habitats in the Mediterranean region. Therefore; it is the first research about lichen community composition subject on maquis habitats in Eastern Mediterranean Region.

Among the frequency and richness data of selected Mediterranean maquis and arborescent matorral habitats from tops and four slope directions of three hills in the northern data were found significantly higher and differ than others. According to results from Sorensen Similarity Index: slopes at same directions are similar, also top localities shows similarity within themselves. Among them northern localities shows the highest similarity. According to MRPP results; species composition of northern slopes is statistically different than southern, eastern, western slopes and top localities. In addition, it was found; all lichen diversity calculation classes (total richness, total frequency, epiphytic and non-epiphytic richness, epiphytic and non-epiphytic frequency) are related with the northern slopes according to NMS ordination test. Hence, it was concluded that all lichen diversity calculation classes are high in the northern localities. North slopes of hills are typically cool, shady and have high atmospheric humidity (Moser et al., 1979). As lichens are poikilohydric organisms, their water content changes according to the surrounding environmental conditions (Nash, 2008). After recurring hydration - drying cycles, they become metabolically active in humid air (Bidussi et al., 2013). In Mediterranean Region when temperature is sufficient for metabolic activity of lichens, humidity becomes more critical than light in terms of vitality (Aragón et al., 2010). Furthermore, dew is a very important water supply for lichens. Dew at Northern slopes has higher frequency and longer duration than the ones at the other directions (Kidron et al., 2002). All this information may be used to explain the reason for the higher similarities and diversities of the northern slopes in Mediterranean maquis and arborescent matorral habitats compared to the other localities observed in this study.

In a previous study, the response of lichen richness and abundance were examined according to land use intensity. According to this study; epilithic lichen diversity increases along the gradient of increase intensity of land use from forested to nonforested areas (Giordani et al., 2010). Similarly, according to NMS ordination test of current study; richness of non-epiphytic lichens, which lives on rocks and ground, was found to associate with the data of the tops of the hills which are the most nonvegetation areas of the Mediterranean maquis and arborescent matorral habitats. Besides, the epiphytic richness was determined to be more related to the southern slopes than other slopes. The most important environmental factor for epiphytic lichens is diffuse but fairly bright light (Rose, 1992; Renhorn et al., 1997; Sillett et al., 2000). This kind of light is mostly on the southern slopes. This may be one of the reasons to explain the relationship between southern slopes and epiphytic lichens in Mediterranean maquis and arborescent matorral habitats.

According to the results obtained from three hills in the Altınbeşik Cave National Park, least amount of lichen species richness but most frequency data were determined 
from İnönü Hill. While species frequency of this hill was found statistically different than other hills, species richness is different than only Altınbeşik Hill. As İnönü hill represents altitudes of $900-1100 \mathrm{~m}$, this finding suggests; it is possible to find interesting lichen composition in Mediterranean maquis and arborescent matorral habitats at these altitudes in comparison to other altitudes.

Also lichen community composition of 3 hills was found similar according to NMS results. In a similar research on macro lichen diversity; the species richness and species abundance of areas were compared (Wiersma and McMullin, 2018). According to this study, it is reported that there is no difference in species richness or abundance in largescale areas. This case suggests that similarity and difference measurements of lichen community compositions may be misleading on large-scale research areas. Similarly in this study, it was found that the composition of lichen in Mediterranean maquis and arborescent matorral habitats can show great variation in small-scale areas.

\section{Conclusion}

Mediterranean maquis and arborescent matorral habitat type is found across the entire Mediterranean biogeographical zone. In this study, it was aimed to determine the epiphytic and non-epiphytic lichen diversity in Mediterranean maquis and arborescent matorral habitats. Lichen diversity in this study was calculated according to the data of species richness and species frequency. In addition, it was examined the existing differences or similarities of lichen diversity at different directions, altitudes, and substrates (epiphytic and non-epiphytic) in this habitat type. Among the frequency and richness data of selected Mediterranean maquis and arborescent matorral habitats from tops and four slope directions of three hills, the northern data were found significantly higher and differ than others. According to results from Sorensen Similarity Index: slopes at same directions are similar, also top localities shows similarity within themselves. Among them northern localities shows the highest similarity. According to MRPP results; species composition of northern slopes is statistically different than southern, eastern, western slopes and top localities. In addition, it was found; all lichen diversity calculation classes (total richness, total frequency, epiphytic and non-epiphytic richness, epiphytic and non-epiphytic frequency) are related with the northern slopes according to NMS ordination test. In addition, richness of non-epiphytic lichens, which lives on rocks and ground, was found to associate with the data of the tops of the hills which are the most non-vegetation areas of the Mediterranean maquis and arborescent matorral habitats. Besides, the epiphytic richness was determined to be more related to the southern slopes than other slopes. Though it was found that lichen community composition shows great variation in Mediterranean maquis and arborescent matorral habitat, community composition of 3 hills was found similar according to NMS results. This result suggests that choosing large-scale areas for determining differences on lichen communities may have misleading results.

\section{REFERENCES}

[1] Aragón, G., Rico, V. J., Belinchón, R. (2006): Lichen diversity from Cazorla, Segura and Las Villas Biosphere Reserve (SE Spain). - Nova Hedwigia 82(1-2): 31-50. 
[2] Aragón, G., Martínez, I., Izquierdo, P., Belinchón, R., Escudero, A. (2010): Effects of forest management on epiphytic lichen diversity in Mediterranean forests. - Applied Vegetation Science 13: 183-194.

[3] Asta, J., Erhardt, W., Ferretti, M., Fornasier, F., Kirschbaum, U. Nimis, P. L., Purvis, O. W., Pirintsos, S., Scheidegger, C., Van Haluwyn, C., Wirth, V. (2002a): European Guideline for Mapping Lichen Diversity as an Indicator of Environmental Stress. The British Lichen Society. - Kluwer Academic Publishers, Dordrecht.

[4] Asta, J., Erhardt, W., Ferretti, M., Fornasier, F., Kirschbaum, U., Nimis, P. L., Purvis, O. W., Pirintsos, S., Scheidegger, C., Van Haluwyn, C., Wirth, V. (2002b): Mapping Lichen Diversity as an Indicator of Environmental Quality. - In: Nimis, P. L., Scheidegger, C., Wolseley, P. A. (eds.) Monitoring with Lichens. Kluwer Academic Publisher, Dordrecht, pp. 273-279.

[5] Bartels, S. F., Chen, H. Y. (2015): Epiphytic macrolichen cover, richness and composition in young successional boreal forest: a comparison of fire and logging disturbance. - Forest Ecology and Management 347: 149-155.

[6] Bidussi, M., Gauslaa, Y., Solhaug, K. A. (2013): Prolonging the hydration and active metabolism from light periods into nights substantially enhances lichen growth. - Planta 237: 1359-1366.

[7] Branquinho, C., Matos, P., Pinho, P. (2015): Lichens as Ecological Indicators to Track Atmospheric Changes: Future Challenges. - In: Lindenmayer, D. B., Pierson, J., Barton, P. (eds.) Surrogates and Indicators in Ecology, Conservation and Environmental Management. CSIRO Publishing and CRC Press, Melbourne and London.

[8] Christensen, S. N., Svane, S. (2007): Contribution to the knowledge of the lichen flora of Crete (Kriti), Greece. - Willdenowia 37(2): 587-593.

[9] Çinbilgel, I., Gökçeoğlu, M. (2010a): The vegetation of Altınbeșik Cavern National Park (İbrad1-Akseki/Antalya-Turkey). A synecological study. - Spanish Journal of Rural Development (SJRD) 1(2): 1-18.

[10] Çinbilgel, I., Gökçeoğlu, M. (2010b): Flora of Altınbeşik Cavern National Park (İbradıAkseki, Antalya/Turkey). - Biological Diversity and Conservation 3: 85-110.

[11] De Guevara, M. L., Lázaro, R., Quero, J. L., Ochoa, V., Gozalo, B. Berdugo, M., Uclés, O., Escolar, C., Maestre, F. T. (2014): Simulated climate change reduced the capacity of lichen-dominated biocrusts to act as carbon sinks in two semi-arid Mediterranean ecosystems. - Biological Conservation 23: 1787-1807.

[12] EEA (2016): European Red List of Habitats - Heathland Habitat Group, F5.1 Mediterranean Maquis and Arborescent Matorral. - EEA Eionet Report.

[13] EEA (2017): EUNIS habitat type hierarchical view revised. Visualization of revised groups of EUNIS habitat type classification - https://eunis.eea.europa.eu/habitats/6121. Accessed: 2019-01-22.

[14] Ellis, C. J., Coppins, B. J., Dawson, T. P., Seaward, M. R. (2007): Response of British lichens to climate change scenarios: Trends and uncertainties in the projected impact for contrasting biogeographic groups. - Biological Conservation 140: 217-235.

[15] Fenn, M. E., Poth, M. A., Aber, J. D., Baron, J. S., Bormann, B. T. Johnson, D. W., Lemly, A. D., McNulty, S. G., Ryan, D. E., Stottlemeyer, R. (1998): Nitrogen excess in North American ecosystems: predisposing factors, ecosystem responses, and management strategies. - Ecological Applications 8: 706-733.

[16] Fryday, A., Coppins, B. (1997): Keys to sterile, crustose saxicolous and terricolous lichens occurring in the British Isles. - Lichenologist 29: 301-332.

[17] Gadsdon, S. R., Dagley, J. R., Wolseley, P. A., Power, S. A. (2010): Relationships between lichen community composition and concentrations of $\mathrm{NO}_{2}$ and $\mathrm{NH}_{3}$. Environmental Pollution 158(8): 2553-2560.

[18] Geiser, L., Neitlich, P. N. (2007): Air pollution and climate gradients in western. Oregon and Washington indicated by epiphytic macrolichens. - Environmental Pollution 145: 203-218. 
[19] Giordani, P., Incert, G., Rizzi, G., Ginaldi, F., Viglione, S. Rellini, I., Modenesi, P. (2010): Land use intensity drives the local variation of lichen diversity in Mediterranean ecosystems sensitive to desertification. - Bibliotheca Lichenologica 105: 139-148.

[20] Giralt, M. (2001): The lichen genera Rinodina und Rinodinella (lichenized Ascomycetes, Physciaceae) in the Iberian Peninsula. - Bibliotheca Lichenologica 79: 1-160.

[21] Gombert, S., Asta, J., Seaward, M. R. D. (2004): Assessment of lichen diversity by index of atmospheric purity (IAP), index of human impact (IHI) and other environmental factors in an urban area (Grenoble, southeast France). - Science of the Total Environment 324: 183-199.

[22] Goward, T., McCune, B., Meidinger, D. (1994): The lichens of British Columbia, illustrated keys. Part 1: Foliose and squamulose species. - British Columbia Ministry of Forests, Special Report Series 8: 1-181.

[23] Johansson, O., Palmqvist, K., Olofsson, J. (2012): Nitrogen deposition drives lichen community changes through differential species responses. - Global Change Biology 18(8): 2626-2635.

[24] Johansson, P. (2008): Consequences of disturbance on epiphytic lichens in boreal and near boreal forests. - Biological Conservation 141: 1933-1944.

[25] John, V. (1996): Preliminary catalogue of lichenised and lichenicolous fungi of Mediterranean Turkey. - Bocconea 6: 173-216.

[26] Kidron, G. J., Herrnstadtw, I., Barzilay, E. (2002): The role of dew as a moisture source for sand microbiotic crusts in the Negev Desert, Israel. - Journal of Arid Environments 52: 517-533.

[27] Koch, N. M., de Azevedo Martins, S. M., Lucheta, F., Müller, S. C. (2013): Functional diversity and traits assembly patterns of lichens as indicators of successional stages in a tropical rainforest. - Ecological Indicators 34: 22-30.

[28] Kruskal, W. H., Wallis, W. A. (1952): Use of ranks in one-criterion variance analysis. Journal of the American Statistical Association 47: 583-621.

[29] Lang, S. I., Cornelissen, J. H., Shaver, G. R., Ahrens, M., Callaghan, T. V., Molau, U., Ter Braak, C. J. F., Hölzer, A., Aerts, R. (2012): Arctic warming on two continents has consistent negative effects on lichen diversity and mixed effects on bryophyte diversity. Global Change Biology 18(3): 1096-1107.

[30] Litterski, B. (1997): Lichen observations on the island of Cyprus. - Feddes Repertorium 108(5-6): 463-473.

[31] McCune, B. (2000): Lichen communities as indicators of forest health. - Bryologist 103: 353-356.

[32] McCune, B. (2016): Key to the lichen Genera of the Pacific Northwest. - Department of Botany and Plant Pathology, Oregon State University, Corvallis OR.

[33] McCune, B., Grace, J., Urban, D. L. (2002): Analysis of Ecological Communities. Gleneden Beach, OR.

[34] McMullin, R. T., Duinker, P. N., Richardson, D. H., Cameron, R. P., Hamilton, D. C., Newmaster, S. G. (2010): Relationships between the structural complexity and lichen community in coniferous forests of southwestern Nova Scotia. - Forest Ecology and Management 260(5): 744-749.

[35] McMurray, J. A., Roberts, D. W., Geiser, L. H. (2015): Epiphytic lichen indication of nitrogen deposition and climate in the northern rocky mountains, USA. - Ecological Indicators 49: 154-161.

[36] Moberg, R. (1977): The lichen genus Physcia and allied genera in Fennoscandia. Symbolae Botanicae Upsaliensis 22: 1-108.

[37] Moser, T. J., Nash, III T. H., Thomson, J. W. (1979): Lichens of Anaktuvuk Pass, Alaska, with emphasis on the impact of caribou grazing. - Bryologist 82: 393-408.

[38] Nascimbene, J., Thor, G., Nimis, P. L. (2012): Habitat types and lichen conservation in the Alps: Perspectives from a case study in the Stelvio National Park (Italy). - Plant Biosystems 146: 428-442. 
[39] Nascimbene, J., Thor, G., Nimis, P. L. (2013): Effects of forest management on epiphytic lichens in temperate deciduous forests of Europe. A review. - Forest Ecology and Management 298: 27-38.

[40] Nash, III T. H. (2008): Lichen Sensitivity to Air Pollution. - In: Nash, III T. H. (ed.) Lichen Biology. Cambridge University Press, Cambridge, UK, pp. 299-314.

[41] Nimis, P. L., John, V. (1998): A contribution to the lichen flora of Mediterranean Turkey. - Cryptogamie. Bryologie, Lichénologie 19: 35-58.

[42] Ravera, S., Nimis, P. L., Brunialti, G., Frati, L., Isocrono, D., Martellos, S., Munzi, S., Nascimbene, J., Potenza, G., Tretiach, M. (2011): The role of lichens in selecting important plant areas in Italy. - Fitosociologia 48(2): 145-153.

[43] Renhorn, K. E., Esseen, P. A., Palmqvist, K., Sundberg, B. (1997): Growth and vitality of epiphytic lichens. I. Responses to microclimate along a forest edge-interior gradient. Oecologia 109: 1-9.

[44] Rogers, P. C., Ryel, R. J. (2008): Lichen community change in response to succession in aspen forests of the Rocky Mountains, USA. - Forest Ecology and Management 256: 1760-1770.

[45] Rose, C. I., Hawksworth, D. L. (1981): Lichen recolonization in London's cleaner air. Nature 289: 289-292.

[46] Rose, F. (1992): Temperate Forest Management: its Effects on Bryophyte and Lichen Floras and Habitats. - In: Bates, J. W., Farmer, A. M. (eds.) Bryophytes and Lichens in a Changing Environment. Clarendon, Oxford, UK, pp. 211-233.

[47] Sillett, S. C., McCune, B., Peck, J. E., Rambo, T. R., Ruchty, A. (2000): Dispersal limitations of epiphytic lichens result in species dependent on old-growth forests. Ecological Applications 10: 789-799.

[48] Sipman, H. J. M., Raus, T. (2015): Lichens and lichenicolous fungi from the island of Chios (Aegean Sea, Greece). - Herzogia 28(2): 496-519.

[49] Smith, C. W., Aptroot, A., Coppins, B. J., Fletcher, A., Gilbert, O. L., James, P. W., Wolseley, P. A. (2009): The Lichens of Great Britain and Ireland. - British Lichen Society, London, UK.

[50] Svoboda, D., Peksa, O., Vesela, J. (2010): Epiphytic lichen diversity in central European oak forests: Assessment of the effects of natural environmental factors and human influences. - Environmental Pollution 158: 812-819.

[51] Thormann, M. N. (2006): Lichens as indicators of forest health in Canada. - The Forestry Chronicle 82: 335-343.

[52] Tomaselli, R. (1977): The degradation of the Mediterranean maquis. - Ambio 356-362.

[53] Tucker, S., Thiers, H. (1998): Key to crustose lichen genera of California. - Bulletin of the California Lichen Society 5: 1-18.

[54] Wasser, S. P., Nevo, E. (2005): Lichen-Forming, Lichenicolous and Allied Fungi of Israel. - Ganter, Ruggell.

[55] Wetmore, C. M. (2005): Keys to the Lichens of Minnesota. - Department of Plant Biology, University of Minnesota, St. Paul.

[56] Wiersma, Y. F., McMullin, R. T. (2018): Is it common to be rare on the landscape? A test using a novel model system. - Landscape Ecology 33: 183-195.

[57] Wirth, V., Hauck, M., Schultz, M. (2013): Die Flechten Deutschlands. - Ulmer, Stuttgart. 